\title{
Diffusion effects in undulator radiation
}

\author{
Ilya Agapov and Gianluca Geloni \\ European XFEL GmbH, Albert-Einstein-Ring 19, 22761 Hamburg, Germany
}

(Received 23 June 2014; published 17 November 2014)

\begin{abstract}
Quantum diffusion effects in undulator radiation in semiclassical approximation are considered. Short-term effects on the electron beam motion are discussed and it is shown that approaches based on diffusion approximation with drift-diffusion coefficients derived from undulator or bending magnet radiation spectrum, and on Poisson statistics with radiation spectrum defined by the local beding field, all lead to similar results in terms of electron energy spread for cases of practical interest. An analytical estimate of the influence of quantum diffusion on the undulator radiation spectrum is derived.
\end{abstract}

DOI: 10.1103/PhysRevSTAB.17.110704

PACS numbers: 41.60.Ap

\section{INTRODUCTION}

Undulator radiation has been long produced experimentally and understood theoretically (see, e.g., [1-4]). Numerical modeling of realistic devices taking realistic field configurations into account has also been long possible [5-7]. In X-ray free electron lasers (FELs) very long undulators are needed so that the Self-amplified spontaneous emission (SASE) process has time to develop. For example, the European XFEL has 22 sections for soft X-ray undulators, and 35 sections for hard X-ray undulators, of approx. $6 \mathrm{~m}$ length each [8]. At such lengths the electron beam diverges significantly and needs focusing. For that reason the undulator comes in segments with focusing magnets and beam instrumentation placed in the intersections. Calculation of spontaneous undulator radiation (SR) from such a setup in an XFEL facility mostly serves diagnostics purposes where two segments are brought in phase by tuning a phase shifter magnet $[9,10]$. SR is also a background signal which is to be taken into account in experiments and X-ray optics design. For most of these purposes rather rough estimates neglecting focusing, emittance and energy spread are typically sufficient. When the required relative precision of SR spectrum calculation is better than 200-300\%, the mentioned effects are to be included. To facilitate future ad-hoc calculations of SR for various purposes, a detailed model of the European XFEL undulator section was introduced in a simulation code [11]. It allows us to calculate radiation spectra taking the energy spread and emittance effects into account. Further discussions of these effects for the parameter space of the European XFEL can be found in [12]. Another effect which is expected to influence the radiation spectrum in such long undulators is

Published by the American Physical Society under the terms of the Creative Commons Attribution 3.0 License. Further distribution of this work must maintain attribution to the author(s) and the published article's title, journal citation, and DOI. the so called quantum diffusion of electron trajectories, and the present paper focuses on this effect.

The effect of electron beam diffusion due to statistical fluctuations in photon emission in the bending magnets of a storage ring has long been known and exploited in synchrotrons, where the beam dimensions are the result of equilibrium between radiative damping and radiative excitations of the betatron and synchrotron oscillations. In this work we focus on the single-pass effects in the undulator radiation, leaving aside the questions of longterm beam stability and equilibrium dimensions, which have already been studied extensively and are receiving further attention in connection to very low emittance storage rings. With the emergence of light sources with large number of insertion devices and the development of free electron lasers with very long undulators, two questions receive attention. First, what are the valid approaches to model the electron trajectories when electron recoil from photon emission is taken into account. And second, how does this influence the undulator radiation spectrum. The first question is studied in Sec. II. We discuss the range of validity of the diffusion approximation, two ways of arriving at the diffusion coefficients, and their respective range of validity. It is shown that for the range of most practical interest, i.e., for the undulator parameter $K \gtrsim 1$, the diffusion approximation with the diffusion coefficients derived from the local field values is adequate, and can be used in calculations of, e.g., radiation integrals [13]. The second question is addressed in Sec. III. Analytical expressions for the influence of energy loss and fluctuations on the undulator radiation spectrum are given. It is shown that for long undulators $\left(L_{w} \gtrsim 10 \mathrm{~m}\right)$, large undulator parameters $[(K \gtrsim 2)]$, and high electron energies $(E \gtrsim 10 \mathrm{GeV}$ ) the effect of quantum diffusion will always be observed. Moreover, for the parameter space of the European XFEL [8], spontaneous undulator radiation intensity at the resonant frequency is reduced by up to an order of magnitude. 


\section{ELECTRON DYNAMICS TAKING RADIATION EMISSION FLUCTUATIONS INTO ACCOUNT}

When calculating synchrotron radiation emitted by electrons in long undulator sections in fields $B \sim 1 \mathrm{~T}$, beam energy distribution dilution caused by quantum fluctuations in the radiated energy has to be taken into account whenever $E \gtrsim 0.5 \mathrm{GeV}$. At the same time, one does not need to consider the quantum nature of the radiation itself unless $\left(\frac{B}{B_{0}}\right) \gamma \sim 1$, where $B_{0}$ is the Schwinger field $B_{0} \sim 4 \times 10^{9} \mathrm{~T}$ and $\gamma$ the relativistic factor [14]. Such conditions exist in astrophysics or in atomic nuclei, but are not yet achieved in accelerators. For synchrotron radiation in particle accelerators, when spin polarization properties of the electrons resulting from radiation emission are not important, it is sufficient to consider radiation computed classically, treating trajectories as random. Computationally, it is most convenient to model the random trajectory by a diffusion process with diffusion-drift coefficients depending on the local magnetic field value. This is however not always possible since the radiation spectrum, from which both the average energy lost by the electron and its fluctuations are derived, cannot be calculated from too short a segment of the trajectory. When the field variation over the radiation formation length is significant, the local approach cannot be, strictly speaking, used. For situations of practical interest where the diffusion starts playing a role, the local approximation is however valid, which is shown in this section. Moreover, expressing the radiated power for a single electron in terms of the number of photons, it can be shown that the average number of photons emitted per undulator period may become less than one. Then one should in principle resort to the more general representation of the electron dynamics by a Poisson process. It is, again, shown, that in cases of practical interest the diffusion approximation remains adequate.

Assume that in time $\Delta t$ the electron loses energy due to (random) emission of radiation quanta. The approach to include the energy loss is to consider an electron moving on a certain trajectory where the emitted spectrum is known, such as a circular path or a sinusoidal path in an undulator, and then calculate the mean and variance of the total radiated power given by that spectrum. For an electron moving on a path with a constant radius of curvature $\rho$ this can be obtained according to [15]. The assumption is that during the time $\Delta t=\rho / \gamma c$ the radius of curvature stays constant (the time needed for the radiation cone to sweep by an angle $1 / \gamma)$. Since

$$
\gamma m_{e}[\mathrm{GeV} / \mathrm{c}]=\rho[\mathrm{m}] \cdot B[\mathrm{~T}] \cdot 0.2998,
$$

the requirement that the path length during this "emission time" is much less than the undulator period

$$
\Delta t \cdot c=\rho / \gamma=m_{e}[\mathrm{GeV}] / 0.2998 B[\mathrm{~T}] \ll l_{w}[\mathrm{~m}]
$$

or roughly

$$
1 / B[\mathrm{~T}] \ll 6 \cdot l_{w}[\mathrm{~cm}] .
$$

Assuming that the energy of each emitted photon and the number of photons emitted are not correlated, and the latter has Poisson statistics [16] with the property $\left\langle N^{2}\right\rangle=\langle N\rangle^{2}+\langle N\rangle$, one arrives at the drift and diffusion coefficients $a(\gamma)$ and $b(\gamma)$ given by

$$
m_{e} c^{2} a(\gamma)=P_{\gamma}, m_{e} c^{2} b^{2}(\gamma)=\frac{55 \sqrt{3}}{72 m_{e} c^{2}} P_{\gamma} u_{c},
$$

where $P_{\gamma}=2 m_{e} c^{3} r_{e} \frac{\gamma^{4}}{\rho^{2}}$ is the average radiation power and $u_{c}=\frac{3}{2} \frac{\hbar \gamma^{3}}{\rho}$ the critical photon energy. Note that substituting the mean magnetic field of a planar undulator into this expression one gets

$$
\frac{\left\langle d(\delta \gamma)^{2}\right\rangle}{d(c t)}=\frac{b^{2}(\gamma)}{c} \sim \frac{\gamma^{4}}{l_{w}^{3}} K^{3} .
$$

The condition (1) is not always fulfilled for XFEL undulators when $K$ is small. For example for the European XFEL, $4 \mathrm{~cm}$ and $6.8 \mathrm{~cm}$ period undulators are used, and it might not be strictly fulfilled when producing very short-wavelength radiation, which corresponds to $K<1$. Following a similar approach, but taking the undulator radiation spectrum as a starting point, in [17] the following approximate expression for diffusion in an ideal undulator field was derived

$$
\frac{\left\langle d(\delta \gamma)^{2}\right\rangle}{d(c t)}=\frac{122 \pi^{3}}{15} \chi_{c} r_{e} \frac{\gamma^{4}}{l_{w}^{3}} K^{3} \times f(K),
$$

where for a planar undulator asymptotically

$$
f(K)=1.20+\frac{1}{K+1.50 K^{2}+0.95 K^{3}} .
$$

For $K \ll 1$ the two approaches lead to different asymptotics, and the approach based on local field value should not be used. For $K>1$ both approaches can be used: although the radiation spectra for undulator and bending magnet radiations are different, the total radiated power and its variation are similar, which leads to similar electron dynamics. For an XFEL $40 \mathrm{~mm}$ and $68 \mathrm{~mm}$ undulators, the diffusion coefficients for different beam energies and $K$ parameters are shown in Fig. 1. Due to the $K^{3}$ scaling of the diffusion coefficient, only strong undulators contribute significantly to diffusion and in numerical calculations the local field approach can be used with a good accuracy. The validity of the diffusion approximation for calculating the rms electron beam dimensions is always justified by virtue of summation over a very large number of electrons in the bunch. 

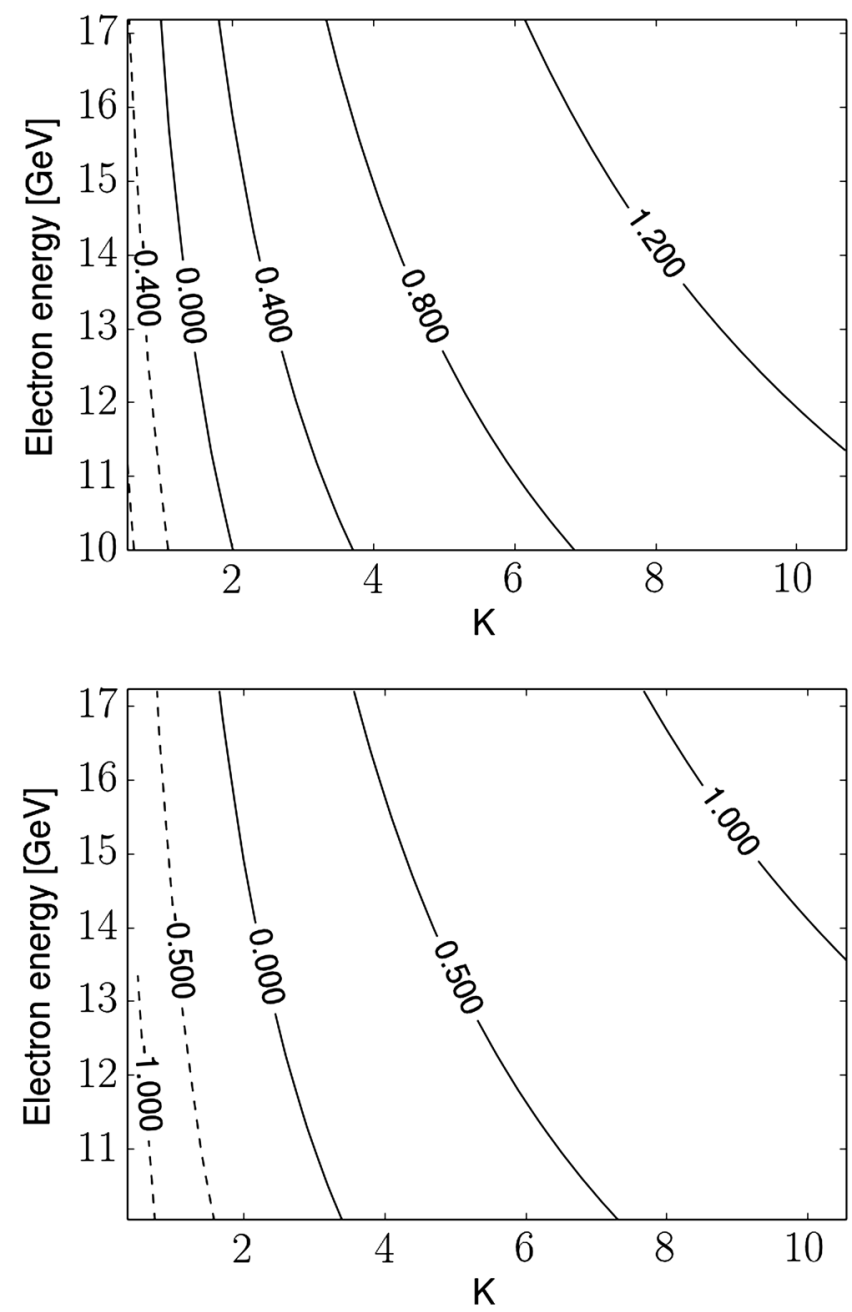

FIG. 1. Diffusion coefficients for SASE XFEL undulators in units of $\log _{10}$ of energy broadening [MeV] per $100 \mathrm{~m}$. The upper plot corresponds to $l_{w}=0.04 \mathrm{~m}$ and the lower to $l_{w}=0.068 \mathrm{~m}$. Dashed lines correspond to negative exponent values.

The validity of the diffusion approximation is further confirmed by numerical Monte-Carlo simulations [18]. These simulations are based on the following method. The bending magnet spectrum is used to calculate the mean free path based on the local radius of curvature of the electron trajectory in the magnetic field. A random time is generated using Poisson statistics, and the electron is tracked for that period of time. After that a photon is generated. The photon carries the momentum directed along the electron path at the emission time and its energy is randomly generated according to the bending magnet spectrum. A transverse photon momentum can be generated based on the radiation opening angle estimate, it has however no noticeable effect and is not used in the simulations. The photon momentum is finally subtracted from the electron momentum and the tracking is continued. More details of the implementation are given in [18]. Example of the electron and photon trajectories resulting from such calculations are shown in Fig. 2.

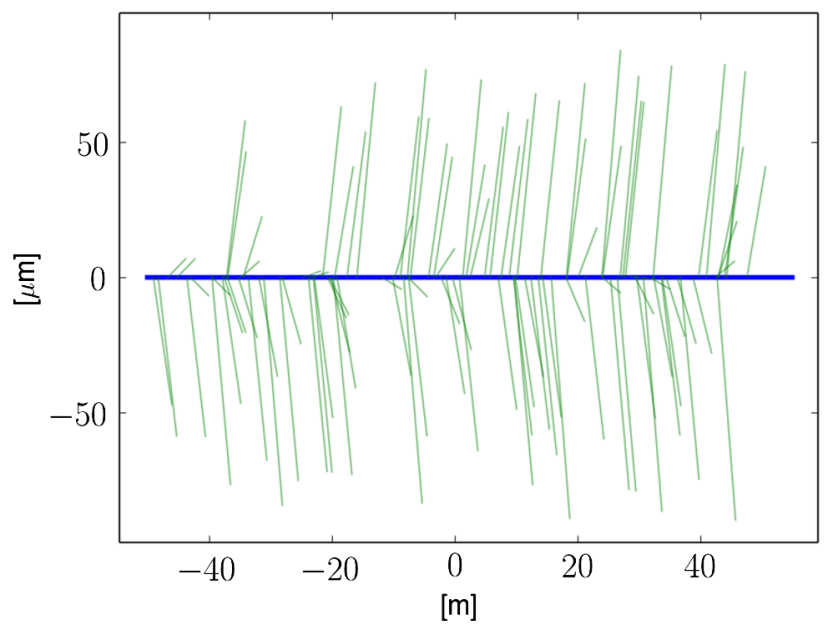

FIG. 2. Electron (blue) and photon (green) trajectories, for $17.5 \mathrm{GeV}$ electron beam, $K=1.0$, over $100 \mathrm{~m}$.

A stochastic leap-frog integrator was also implemented. In this integrator a normally distributed value is added to the electron energy at equal time steps. The mean and variance of this value corresponds to the drift and diffusion coefficients (2) or (3). For cases of practical interest $(K>1)$, all these approaches lead to similar results for the resulting energy spread. Example of comparison of the Monte-Carlo method to the stochastic integrator are shown in Fig. 3.

Although leading to correct beam energy spread, the diffusion approximation can lead to unphysical single trajectories in the longitudinal phase space, since the electron energy can grow, with a certain probability, as a result of photon emission. Since in both bending magnet and undulator radiation the characteristic energy of emitted photons is much smaller than the electron energy, many

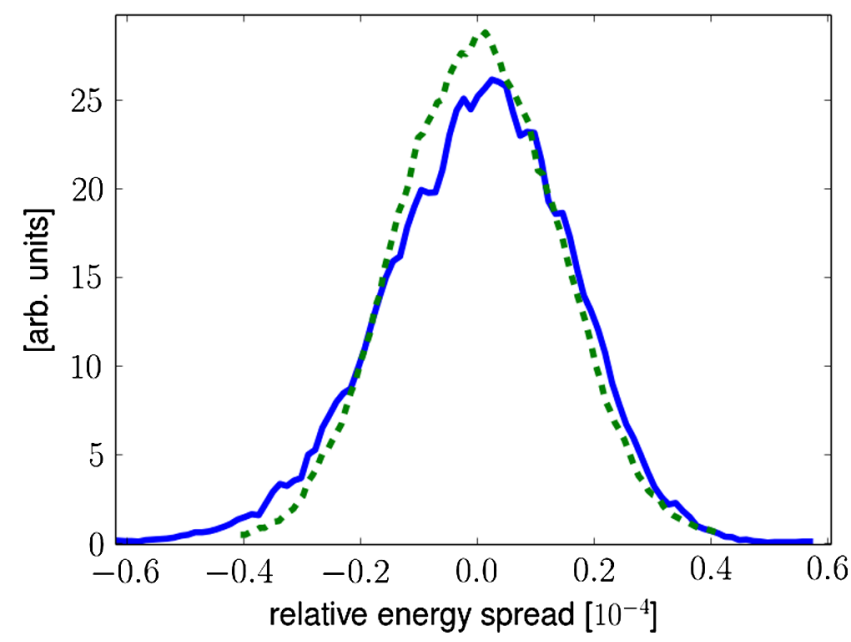

FIG. 3. Energy spread $\gamma / \gamma_{0}$ at the undulator exit, from initially delta-distributed beam. Direct Monte Carlo (solid) and stochastic integration [dashed, diffusion coefficient corresponding to Eq. (3)], for $17.5 \mathrm{GeV}$ electron beam, $K=1.0$, over $100 \mathrm{~m}$. 
such emissions need to occur before the electron changes energy sufficiently. Thus, in most practical situations the small deviations of single trajectories from those resulting from Poisson statistics can be neglected.

One practical consequence of the results discussed in this section is that whenever diffusion is not negligible, the diffusion coefficient is to a good approximation given by the local magnetic field along the electron trajectory. This means that expressions for the equilibrium beam dimensions in storage rings where integrals involving local radius of curvature enter [13] are valid for the undulator case too.

\section{EMITTED SPECTRA IN THE DIFFUSION APPROXIMATION}

The standard derivation of the undulator radiation spectrum in frequency domain based on paraxial version of Maxwell's equation (see, e.g., [19]) allows for extevmnsion to compute the statistical moments. In the paraxial approximation, the Fourier component of the radiation field at observation point $\mathbf{r}_{\perp 0}, z_{0}$ is

$$
\tilde{\mathbf{E}}_{\omega}=-\frac{i \omega e}{4 \pi c^{2}} \int_{-L}^{L} d z \frac{e^{i \phi}}{z_{O}-z}\left[\boldsymbol{\beta}(z)-\frac{\mathbf{r}_{\perp 0}-\mathbf{r}_{\perp}(z)}{z_{0}-z}\right],
$$

where

$$
\phi=\omega\left[\frac{s(z)}{v}-\frac{z}{c}+\frac{\left|\mathbf{r}_{\perp 0}-\mathbf{r}_{\perp}\right|^{2}}{2 c\left(z_{0}-z\right)}\right] .
$$

Here $\mathbf{r}_{\perp}(z)$ and $\boldsymbol{\beta}(z)$ are the position and relativistic velocity of the electron, and $s(z)$ its path length as a function of the longitudinal coordinate. In a planar undulator the trajectory is to a good accuracy

$$
\begin{aligned}
x(z) & =\frac{K}{\gamma k_{w}} \cos \left(k_{w} z\right), \\
\boldsymbol{\beta}(z) & =-\frac{K}{\gamma} \sin \left(k_{w} z\right), \\
s(z) & =\frac{\beta}{\beta_{z}} z-\frac{K^{2}}{8 \gamma^{2} k_{w}} \sin \left(2 k_{w} z\right), \\
\beta_{z} & =\beta\left(1-\frac{K^{2}}{4 \gamma^{2}}\right),
\end{aligned}
$$

where the undulator wave number $k_{w}=2 \pi / l_{w}$ was used. In the following we perform estimates for on-axis spectrum only, which is sufficient for our purposes. Analysis of offaxis spectrum or spectrum integrated over a finite aperture is more technically involved and better done numerically, but the qualitative estimates of diffusion effects still hold. Moreover, it is assumed that the number of undulator periods $N_{w}$ is large and the observation point is in far zone. Then only polarization component in the plane of motion is considered, which is

$$
\begin{aligned}
\tilde{\mathbf{E}}_{\omega, x} & =-\frac{i \omega e}{4 \pi c^{2} z_{0}} \int_{-L}^{L} \frac{K}{\gamma} \sin \left(k_{w} z\right) e^{i \phi} d z \\
& \equiv i C_{1} \int_{-L}^{L} \frac{1}{\gamma} \sin \left(k_{w} z\right) e^{i \phi} d z, \\
\phi & =\frac{\omega}{\omega_{1}}\left[k_{w} z-\frac{K^{2} \omega_{1}}{8 \gamma^{2} k_{w} c} \sin \left(2 k_{w} z\right)\right], \\
\omega_{1}^{-1} & =\frac{1}{2 k_{w} c \gamma^{2}}\left(1+\frac{K^{2}}{2}\right),
\end{aligned}
$$

where $L=N_{w} l_{w} / 2$ is the undulator half-length. The integral is evaluated with the help of the identity

$$
e^{i u \sin \psi}=\sum_{n=-\infty}^{\infty} J_{n}(u) e^{i n \psi},
$$

where $J_{n}$ are Bessel functions of first kind

$$
\begin{aligned}
\tilde{\mathbf{E}}_{\omega, x} & =i C_{1} \sum_{n=-\infty}^{\infty} \int_{-L}^{L} \frac{1}{\gamma} J_{n}(u) e^{i k_{w} z\left(\frac{\omega}{\omega_{1}}+2 n\right)} \sin \left(k_{w} z\right) d z \\
& =C_{1} \sum_{n=-\infty}^{\infty} \int_{-L}^{L} \frac{1}{2 \gamma} J_{n}(u)\left[\sin \Psi_{n,+} z-\sin \Psi_{n,-} z+i\left(\cos \Psi_{n,-} z-\cos \Psi_{n,+} z\right)\right] d z \\
\Psi_{n, \pm} & =k_{w}\left(\frac{\omega}{\omega_{1}}+2 n \pm 1\right), \quad u=\frac{\omega}{\omega_{1}} \frac{K^{2}}{4\left(1+\frac{K^{2}}{2}\right)}=\frac{\omega K^{2}}{8 k_{\omega} c \gamma^{2}}
\end{aligned}
$$

If $\gamma$ and thus $u$ and $\omega_{1}$ are assumed constant, only cosine terms contribute to the integral and this becomes

$$
\tilde{\mathbf{E}}_{\omega, x}=-\frac{C_{1} L}{\gamma} \sum_{n=-\infty}^{\infty} J_{n}(u)\left(\frac{\sin \Psi_{n,+} L}{\Psi_{n,+} L}-\frac{\sin \Psi_{n,-} L}{\Psi_{n,-} L}\right) .
$$


This expression has resonant behavior around $\omega=(-2 n \pm 1) \omega_{1}$, e.g., for the first harmonic one is left with two terms

$$
\begin{aligned}
\tilde{\mathbf{E}}_{\omega, x} & =-\frac{C_{1} L}{\gamma}\left[J_{0}(u)-J_{1}(u)\right] \frac{\sin \Psi_{0,+} L}{\Psi_{n,+} L} \\
& =-\frac{\omega e K N_{w} l_{w}}{4 \pi \gamma c^{2} z_{0}}\left[J_{0}(u)-J_{1}(u)\right] \frac{\sin \pi N_{w} \Delta \omega / \omega_{1}}{\pi N_{w} \Delta \omega / \omega_{1}},
\end{aligned}
$$

where $\Delta \omega=\omega-\omega_{1}$. Now if the change of energy is taken into account, $J_{n}(u)$ and $1 / \gamma$ cannot be taken out of the integral. The contribution of the phase and amplitude factors to the integral can be estimated separately. Assuming the phase constant, $J_{n}(u)$ and $1 / \gamma$ can be developed into Taylor series taking only the linear part of the energy change $\delta \gamma(t)$. To estimate the amplitude first note that the integrals of the type

$$
I=\int_{-L}^{L} \delta \gamma(z) f(z) d z
$$

where $\delta \gamma(z)$ is a random walk type process with $\langle\delta \gamma(-L)\rangle=0$ and independent Gaussian increments, have moments

$$
\begin{aligned}
\langle I\rangle & =a \int_{-L}^{L} t f(t) d t,\left\langle I^{2}\right\rangle-\langle I\rangle^{2} \\
& =b^{2} \int_{-L}^{L} d s\left[\int_{s}^{L} f(t) d t\right]^{2} .
\end{aligned}
$$

Using $\frac{1}{\gamma} \approx \frac{1}{\gamma_{0}}-\frac{1}{\gamma_{0}^{2}} \delta \gamma$ and taking into account approximations $J_{0}(u) \approx 1-\frac{u^{2}}{4}, J_{1}(u) \approx \frac{u}{2}$ (for $u<1$ ) one sees that the contributions to the integral (4) from the variation of $J(u)$ and $\gamma(z)$ are of order $1 / \gamma$ and can be neglected. The major contribution is in the phase since $\Psi$ becomes a function of $z$ and the phase shift in the integrand is of order $k_{w} L \delta \omega / \omega_{1}$ which is not small. This is in analogy with incoherent summation of fields from independent emitters. Now consider the phase only, and consider the contribution of energy drift term. Up to the first order in $\delta \gamma$ the integrand phase for the first harmonic becomes

$$
\Psi_{1}=k_{w}\left(\frac{\Delta \omega}{\omega_{1}}+2 \frac{\omega}{\omega_{1}} \delta \gamma\right)=k_{w}\left(\frac{\Delta \omega}{\omega_{1}}+2 \frac{\omega}{\omega_{1}} \hat{a} z\right),
$$

where $\hat{a}=\frac{2 P_{\gamma}}{m_{\rho} c}$ is the relative energy loss per meter. Integration can be done with the help of Fresnel integrals $C$ and $S$

$$
\begin{aligned}
\tilde{\mathbf{E}}_{\omega, x}= & -\frac{\omega e K}{4 \pi \gamma c^{2} z_{0}}\left[J_{0}(u)-J_{1}(u)\right] \frac{\sqrt{\pi}}{\sqrt{2 \hat{a} k_{w}}} \\
& \times\left(\cos \frac{k_{w}\left(\Delta \omega / \omega_{1}\right)^{2}}{4 \hat{a}}\left\{C\left[\frac{\sqrt{k_{w}}\left(\Delta \omega / \omega_{1}+N_{w} l_{w} \hat{a}\right)}{\sqrt{2 \pi \hat{a}}}\right]-C\left[\frac{\sqrt{k_{w}}\left(\Delta \omega / \omega_{1}-N_{w} l_{w} \hat{a}\right)}{\sqrt{2 \pi \hat{a}}}\right]\right\}\right. \\
& \left.+\sin \frac{k_{w}\left(\Delta \omega / \omega_{1}\right)^{2}}{4 \hat{a}}\left\{S\left[\frac{\sqrt{k_{w}}\left(\Delta \omega / \omega_{1}+N_{w} l_{w} \hat{a}\right)}{\sqrt{2 \pi \hat{a}}}\right]-S\left[\frac{\sqrt{k_{w}}\left(\Delta \omega / \omega_{1}-N_{w} l_{w} \hat{a}\right)}{\sqrt{2 \pi \hat{a}}}\right]\right\}\right) .
\end{aligned}
$$

Numerical values of the intensity for various values of $\hat{a}$ are plotted in Fig. 4. The energy loss of electrons can be compensated by undulator tapering, i.e., changing $K$ so that $\omega / \omega_{1}$ is kept constant. Then the effect of energy loss becomes negligible again. Note that this argument applies to the radiation at an arbitrary angle $\theta$, where the resonant frequency is given by

$$
\omega_{1}^{-1}=\frac{1}{2 k_{w} c \gamma^{2}}\left(1+\frac{K^{2}}{2}+\gamma^{2} \theta^{2}\right)
$$

but does not apply to the radiation integrated over a finite solid angle.

Fluctuations in the phase factor cannot be compensated with tapering since they vary for each electron independently. It is possible to estimate the spectrum in the limit of small or large fluctuations (in the first case, representing the term proportional to $e^{i z^{2}}$ by its linear part, in the latter by

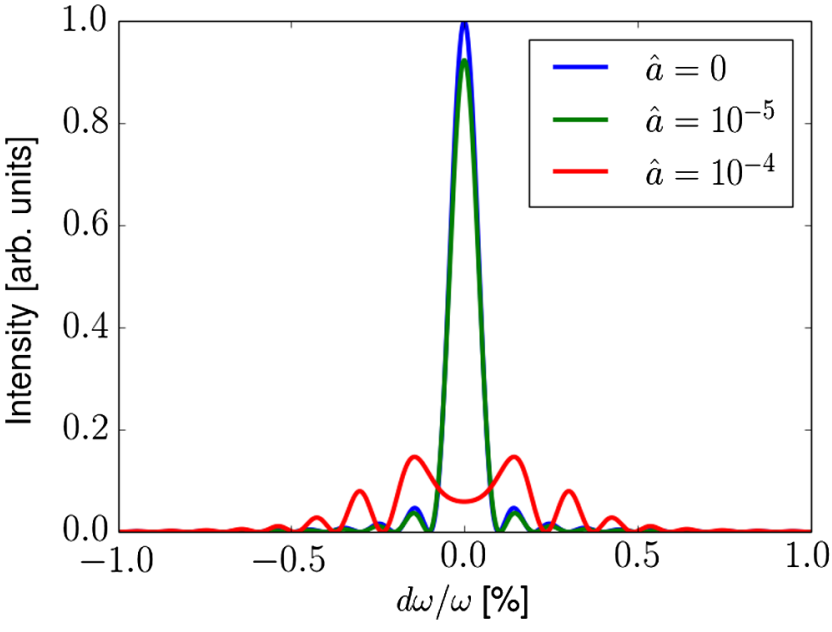

FIG. 4. Spectrum corresponding to various energy drift parameters, $N_{w}=1000$. 


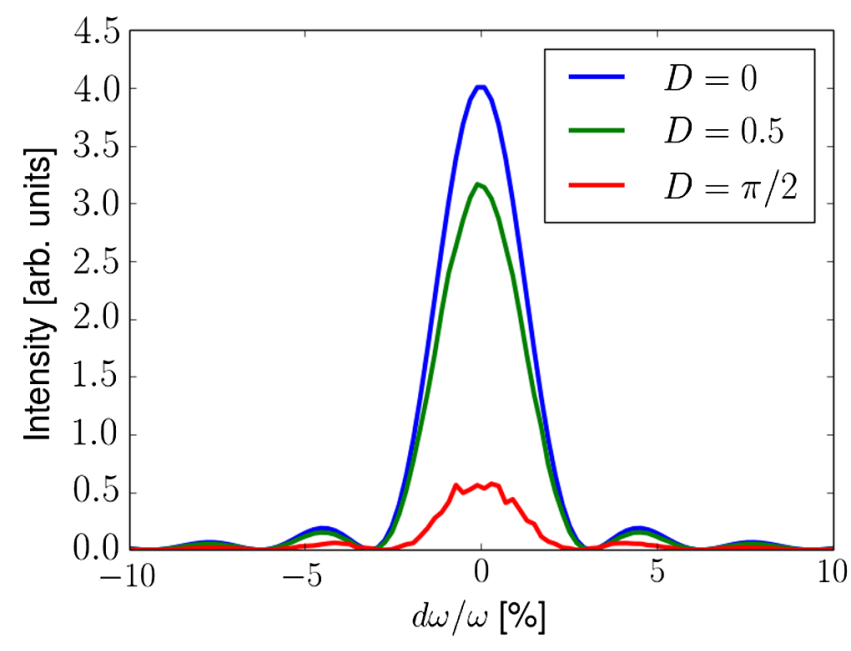

FIG. 5. Spectrum corresponding to various diffusion dephasing parameters, averaged over 1000 realizations. $N_{w}=100$.

representing the integral as a sum of terms with uncorrelated phases). In practice the phase factor arising in fluctuations need not be small or large, which is the case whenever $N_{w}(\delta \gamma / \gamma)_{z} \sim 1$, where $(\delta \gamma / \gamma)_{z}$ is the rms diffusive energy spread at distance $z$, e.g., at the undulator exit. It can be calculated with the methods of Sec. II. This happens for some part of the European XFEL parameter space (see Fig. 1). To understand the spectrum in this case it is easier to introduce the "diffusive dephasing" parameter

$$
D=4 \pi N_{w}(\delta \gamma / \gamma)_{z}
$$

The dependency of the undulator radiation spectrum on the diffusive dephasing parameter is shown in Fig. 5. The effect will be noticeable at the European XFEL for all wavelengths. For example for the soft X-ray undulator at $17.5 \mathrm{GeV}$ in the radiation wavelength range $\sim 1 \mathrm{KeV}$ $(K \sim 7)$, the diffusive energy spread will be $\sim 5 \mathrm{MeV}$, which taking into account $N_{w} \approx 2000$ results in $D=2 \pi$. In that case the peak spectral intensity for the on-axis radiation is reduced by more than an order of magnitude.

Note that the on-axis spontaneous radiation spectrum can be more sensitive to the emission fluctuations than the SASE FEL radiation [20]. This can be explained so that in a SASE FEL for the exponential power growth regime only dephasing effects over one gain length play a role, the latter being only a fraction of the full undulator length.

\section{CONCLUSION}

We discussed approaches to calculating the electron beam energy spread due to quantum diffusion, and showed that for cases of practical interest the diffusion coefficients can be calculated based on the local magnetic field, which greatly simplifies several types of calculations as compared to using the diffusion coefficients obtained from the undulator radiation spectrum. The effects of Poisson statistics in the photon emission was shown to be negligible.

In synchrotron light sources where single undulator length is typically a few meters and the energies are a few $\mathrm{GeV}$, the effect of quantum diffusion enters the undulator radiation spectrum indirectly by influencing the equilibrium electron beam parameters [21], while its single-pass effect on the radiation can be neglected. This is not the case for FEL undulators. It has been shown that quantum diffusion results in significant spectrum broadening. This has to be taken into account whenever spontaneous synchrotron radiation from long undulators is studied. The influence of the diffusion effect on the spectrum can be estimated with the dephasing parameter.

\section{ACKNOWLEDGMENTS}

The authors would like to thank O. Chubar, M. Scheer, and E. Saldin for useful discussions.

[1] H. Motz, J. Appl. Phys. 22, 527 (1951).

[2] V. L. Ginzburg, Izv. AN SSSR. Ser. Fiz. 11, 165 (1947).

[3] D. F. Alferov, Yu. A. Bashmakov, and E. G. Bessonov, Tr. FIAN SSSR 80, 100 (1975).

[4] A. Hofmann, The Physics of Synchrotron Radiation (Cambridge University Press, Cambridge, England, 2004).

[5] O. Chubar and P. Elleaume, in Proceedings of the 6th European Particle Accelerator Conference, Stockholm, 1998 (IOP, London, 1998).

[6] M. Scheer, in Proceedings of the 11th International Computational Accelerator Physics Conference, ICAP-2012, Rostock-Warnemünde, Germany (JACoW, Rostock-Warnemünde, 2012).

[7] T. Tanaka and H. Kitamura, J. Synchrotron Radiat. 8, 1221 (2001).

[8] M. Altarelli et al., Report No. DESY-2006-097, 2007.

[9] M. Tischer, P. Ilinski, U. Hahn, J. Pflüger, and H. SchulteSchrepping, Nucl. Instrum. Methods Phys. Res., Sect. A 483, 418 (2002).

[10] T. Tanaka et al., Phys. Rev. ST-Accel. Beams 15, 110701 (2012).

[11] I. Agapov et al., Nucl. Instrum. Methods Phys. Res., Sec. A (unpublished).

[12] I. Agapov et al., in Proceedings of the 4th International Particle Accelerator Conference, IPAC-2013, Shanghai, China, 2013 (JACoW, Shanghai, China, 2013).

[13] H. Wiedemann, in Handbook of Accelerator Physics and Engineering, edited by A. Chao and M. Tigner (World Scientific, Singapore, 1999).

[14] A. A. Sokolov and I. M. Ternov, in Radiation from Relativistic Electrons, American Institute of Physics Translation Series (AIP, New York, 1986).

[15] M. Sands, Report No. SLAC-R-121, 1970.

[16] C. Itzykson and J.-B. Zuber, Quantum Field Theory (McGraw-Hill, New York, 1980).

[17] E. L. Saldin, E. A. Schneidmiller, and M. V. Yurkov, Nucl. Instrum. Methods Phys. Res., Sect. A 381, 545 (1996). 
[18] I. Agapov and G. Geloni, in Proceedings of the 4th International Particle Accelerator Conference, IPAC2013, Shanghai, China, 2013 (JACoW, Shanghai, China, 2013).

[19] G. Geloni et al., Report No. DESY-05-032, 2005.
[20] J. Rossbach, E. L. Saldin, E. A. Schneidmiller, and M. V. Yurkov, Nucl. Instrum. Methods Phys. Res., Sect. A 393, 152 (1997).

[21] E. Tarazona and P. Elleaume, Rev. Sci. Instrum. 66, 1974 (1995). 